\title{
Exogenous lipoid pneumonia
}

\author{
DAMIAN J. CORNACCHIA, DO \\ CHRISTOPHER H. SNYDER, DO \\ DANIEL C. DUPONT, DO \\ N. SUSAN YARON, MD
}

\section{Although a rare form of nonresolving pulmonary infiltrate, exogenous lipoid pneumonia is a great mimicker. It often is mistaken for bacterial pneumonia or cancer. Many cases have been diagnosed only by open lung biopsy or other invasive procedures. Depending on the type of lipid ingested and the degree of inflammation that occurs, damage to the lung can be little to none or can fulminate to necrosis and hemorrhage. \\ Symptoms may range from none to respiratory failure. In the case presented, the patient was ingesting Vaseline Intensive Care Lotion and baby oil as laxatives. This information was elicited only after diagnosis was made by open lung biopsy.}

Exogenous lipoid pneumonia often mimicks other pulmonary diseases, including pneumonia and lung cancer. Symptoms can range from occasional cough to severe, debilitating dyspnea and pulmonary illness, occasionally ending in death. Usually, the history of oil ingestion is elicited only retrospectively, and often an invasive procedure is required for definitive diagnosis. In the case reported here, open lung biopsy was the ultimate diagnostic procedure, and only then did the patient admit to the ingestion of baby oil and Vaseline Intensive Care Lotion.

\section{Report of case}

A 34-year-old man was admitted with severe anorexia, nausea, and bowel incontinence of three days' duration. These symptoms were accompanied by lethargy and mental status changes. The patient's medical history included migraine, hypertension, epilepsy, and multiple attempts at suicide. He also was known to have schizophrenia, but he was not institutionalized at the time of admission. His medications included clonidine, triamterene with hydrochlorothiazide, phenytoin, secobarbital sodium, morphine sulfate, metoclopramide hydrochloride, lactulose, and thiothixene.

The patient's temperature was $99.2^{\circ} \mathrm{F}$, and his blood pressure, heart rate, and respiratory rate were stable. His pupils were sluggish. No adenopathy was palpated in the neck. The heart had a regular rhythm, without murmurs, and the lungs were clear bilaterally. Findings of abdominal and extremity examinations were essentially normal. Rectal examination revealed occult blood.

Initial chest roentgenography showed partially consolidated infiltrates and pleural reaction involving the posterior division of the right upper lobe. Bilateral lower lobe infiltrates without pleural effusion also were seen (Fig 1). These findings had not been present one year prior to admission.

Complete blood cell count findings were as follows: WBC, 20,000/cu mm; differential count, 59 segmented neutrophils, 32 band forms, 3 lymphocytes, 1 monocyte, and 5 metamyelocytes; platelet count, $1,326.00 / \mathrm{cu} \mathrm{mm}$; hemoglobin, $8.3 \mathrm{~g} / \mathrm{dL}$; and hematocrit, $27.7 \%$. Metabolic values include the following: sodium, $131 \mathrm{mEq} / \mathrm{L}$; potassium, $2.7 \mathrm{mEq} / \mathrm{L}$; chloride, $90 \mathrm{mEq} / \mathrm{L}$; total $\mathrm{CO}_{2}, 28 \mathrm{mEq} /$ $\mathrm{L}$; glucose, $273 \mathrm{mg} / \mathrm{dL}$; and creatinine, $1.3 \mathrm{mg} / \mathrm{dL}$. Arterial blood gas values (room air) were as follows: $\mathrm{pH}, 7.55$; $\mathrm{pCO}_{2}, 37.6 \mathrm{~mm} \mathrm{Hg} ; \mathrm{pO}_{2}, 61.2 \mathrm{~mm} \mathrm{Hg}$; bicarbonate ion, $33.8 \mathrm{mEq} / \mathrm{L}$; and saturation, $94 \%$.

Hydration procedures were carried out, and a regimen that included the patient's outpatient medications as well as intravenous cefoxitin ( $2 \mathrm{~g}$ every six hours) was begun. The lungs remained clear, and initial sputum cultures grew normal flora and numerous Candida albicans organisms.

On the second hospital day, the patient's temperature was $101.2^{\circ} \mathrm{F}$. Scattered rhonchi were now detected bilaterally and erythromycin (500 mg every six hours) was added to the drug therapy. Blood culturing had negative findings. Titration for Legionella (acute and convalescent stages), cold agglutinins, and HTLV-III had negative findings, as did fluorescent staining.

On the sixth hospital day, repeat chest roentgenography revealed worsening consolidation of infiltrates (Fig 2).

Gram's staining of sputum samples on the seventh hospital day showed numerous fungal mycelia and budding yeast cells, as well as moderate gram-positive cocci in pairs, chains, and clusters.

The drug regimen was modified on the eighth hospital day: The cefoxitin was discontinued, and tobramycin ( 60 mg every 8 hours) and piperacillin ( 3 g every 4 hours) were added. Bronchofibroscopy, bronchoalveolar lavage, and endobronchial biopsy revealed diffuse erythema of the airways and moderate secretions. Cytologic evaluation revealed light growth of Candida albicans, many WBCs, and no evidence of malignancy. Biopsy specimens 


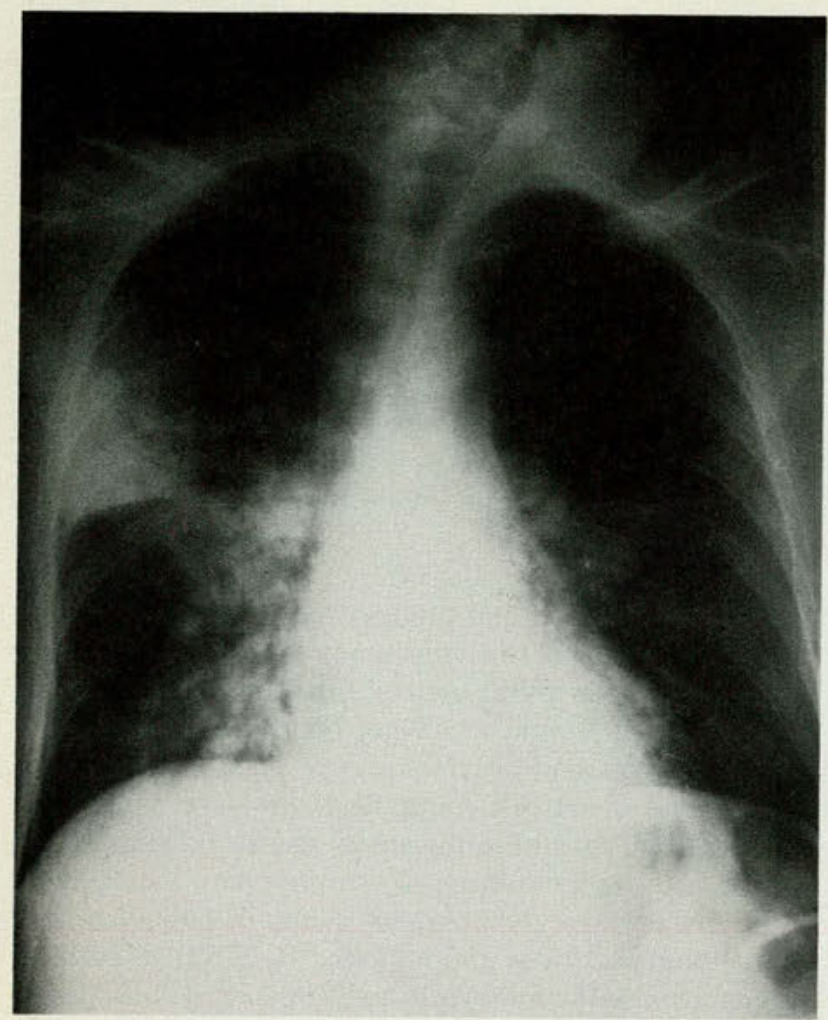

Figure 1. Admission chest film (anteroposterior view) demonstrates partially consolidated infiltrates with pleural reaction in right upper lobe (posterior division). Bilateral lower lobe pneumonia (without pleural effusion) also is present.

revealed lung parenchyma with noninvasive budding yeast cells and interstitial pneumonia.

The patient's condition did not respond to the antibiotic therapy, and all antibiotics were discontinued except for erythromycin, which was continued to combat possible atypical pneumonia. Also, the antifungal agent amphotericin B was added to the drug regimen. Again, there was no clinical response.

Open lung biopsy was performed on the 13th hospital day. Microscopic study showed irregular nodules of lipidladen macrophages and fibrous reaction involving septa and adjacent pleura (Fig 3). High-power magnification revealed large, coalescing 'vacuoles with nuclei pushed to the periphery of the cells, and surrounding macrophages with smaller vacoules (Fig 4).

Nonspecific, lymphoplasmacytic, chronic inflammatory infiltrate also was seen. These findings were consistent with lipoid pneumonia secondary to exogenous ingestion of oil. On questioning, the patient admitted to daily ingestion of Vaseline Intensive Care Lotion and baby oil for the purpose of laxation.

Postoperatively, nosocomial staphylococcal pneumonia developed, and ticarcillin with clavulanate potassium therapy ( 3.1 g every 6 hours) was begun. The eryth-

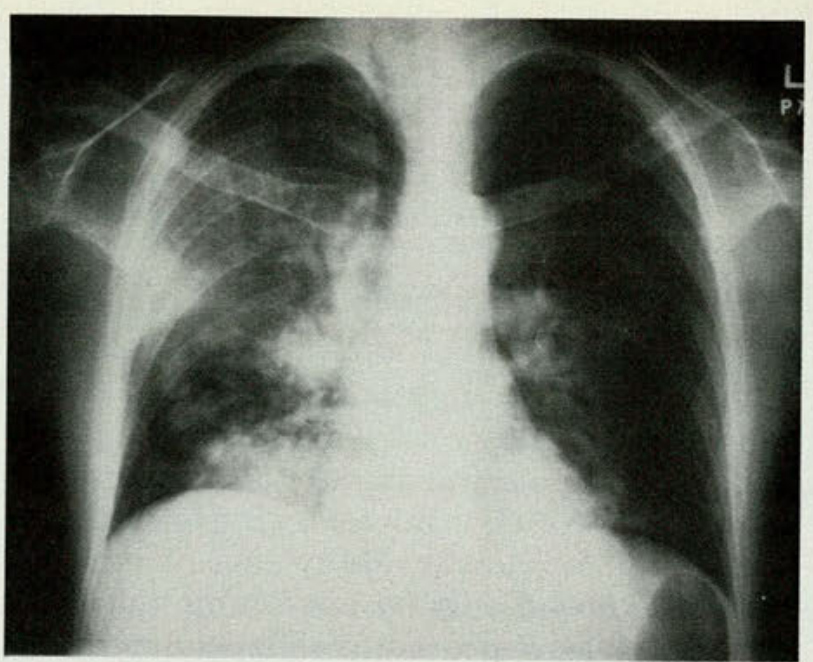

Figure 2. Chest film obtained on sixth hospital day demonstrates worsening consolidation of infiltrates in the right upper lobe and right lower lobe.

romycin therapy also was continued. The patient improved gradually, and he was discharged on the 40th hospital day.

\section{Discussion}

Investigation of exogenous lipoid pneumonia dates back to the $1920 \mathrm{~s} .{ }^{1}$ However, the disease probably has been present and has been the cause of misdiagnosis of illness refractory to usual medical care for centuries. Lately, because of increased awareness and historic background, more cases have been reported. The abuse of laxatives ${ }^{2}$ as well as association with occupation ${ }^{3}$ are seen more frequently in these cases.

Exogenous lipoid pneumonia remains a disorder that should be included in the differential diagnosis, especially when nonresolving, atypical pneumonia seems apparent.

\section{Presentation and progression}

In most cases of exogenous lipoid pneumonia, symptoms are not manifested in a fashion similar to infectious pneumonia. In elderly persons, dyspnea often is the first symptom; as the illness progresses, cough, fever, and chills may be present. Younger patients often have only symptoms of cough and lower respiratory tract infection, or they may have an abnormal chest $\mathrm{x}$-ray on routine evaluation.

Because of the clinician's low suspicion or lack of awareness, a history of aspiration of oil or a related substance is not elicited. A patient who is at risk for aspiration or who has a history of laxa- 


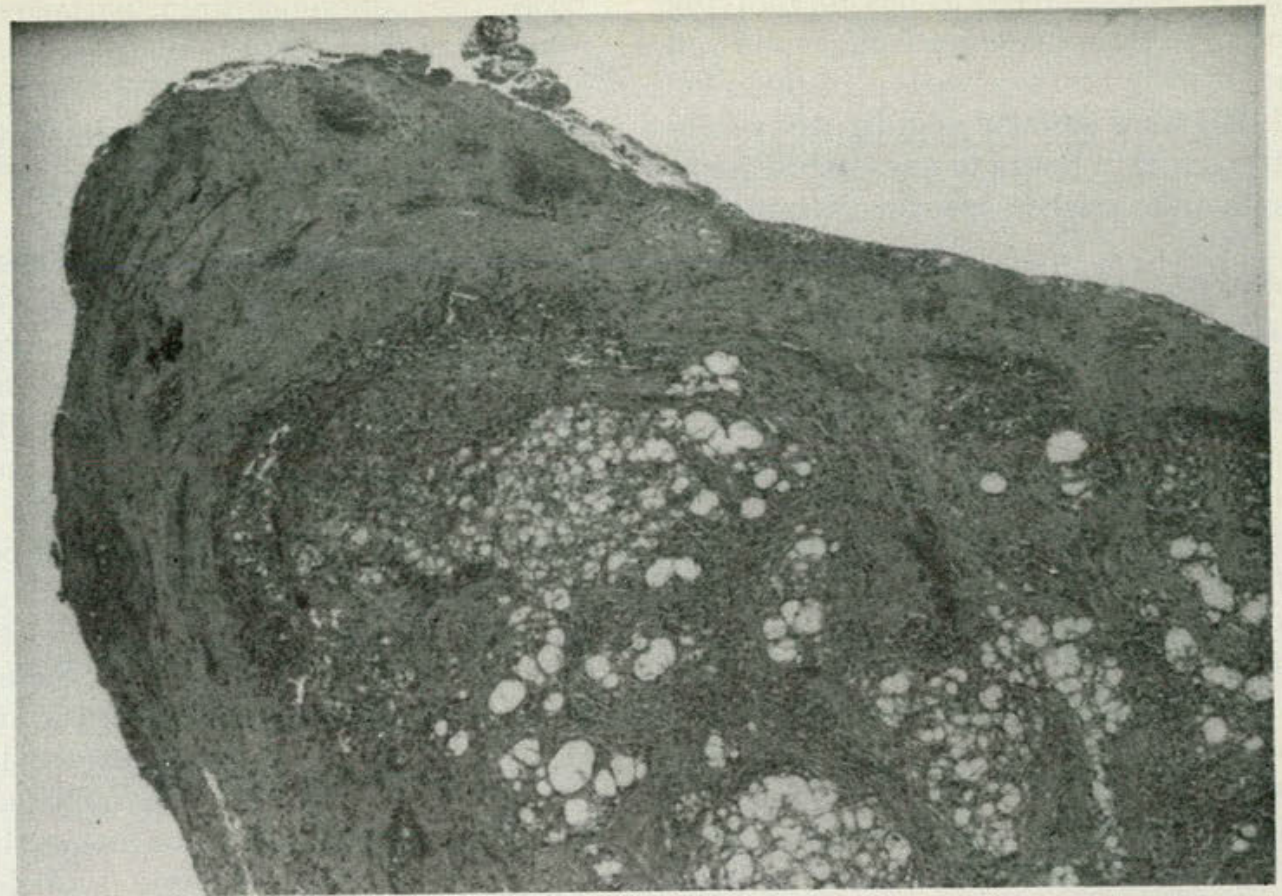

Figure 3. Section of lung shows irregular nodules of lipid-laden macrophages and fibrous reaction involving septa and adjacent pleura. Normal parenchymal architecture is obliterated (hematoxylin and eosin stain, original magnification $\times 40$ ).

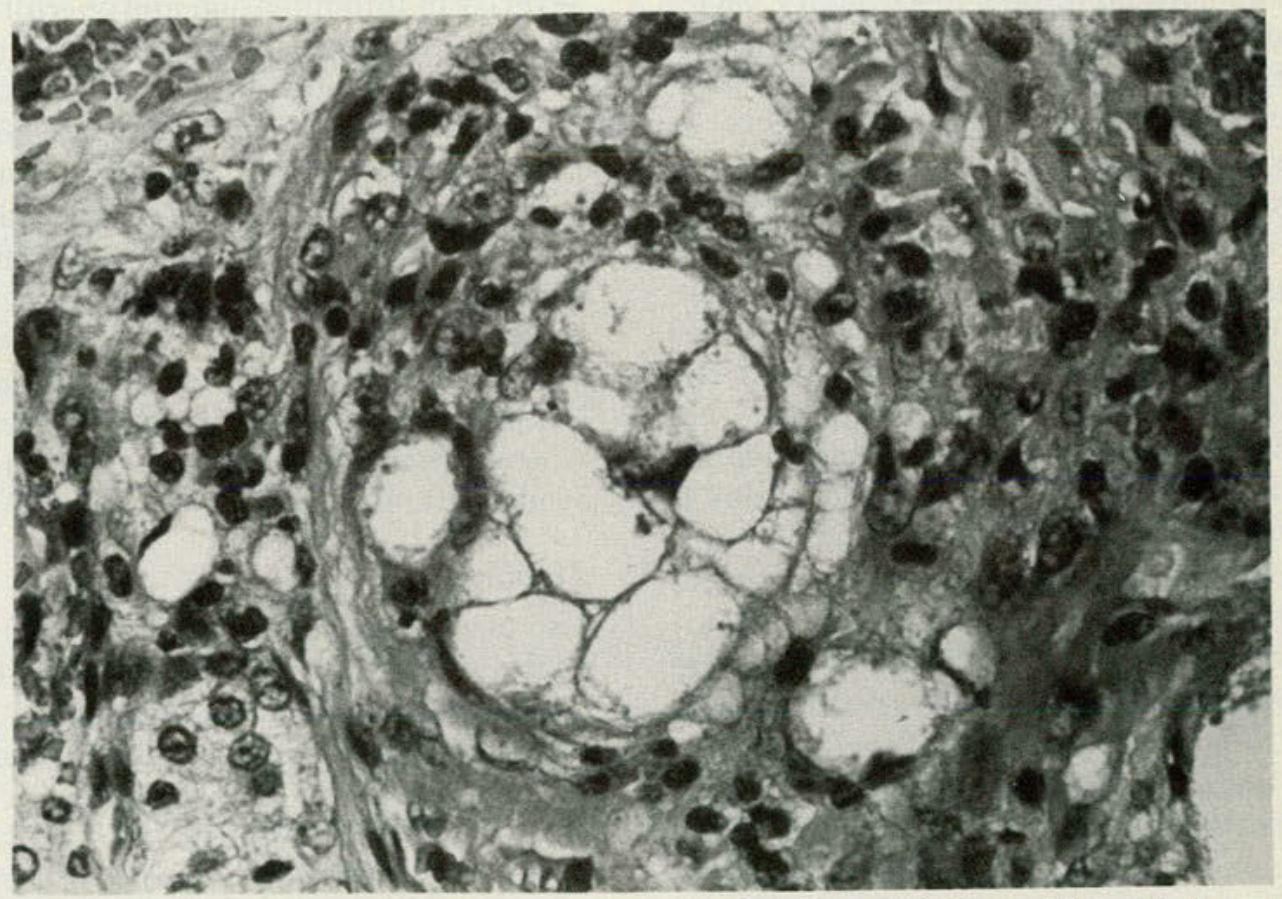

Figure 4. High-power view of large, coalescing vacuoles. Nuclei are pushed to periphery of cells, and surrounding macrophages have smaller vacuoles. Nonspecific lymphoplasmacytic, chronic inflammatory infiltrate is present (hematoxylin and eosin stain, original magnification $\times 400$ ). 
tive need should be considered as possibly having this condition. The time sequence from aspiration to appearance of symptoms varies according to the type of lipid. Higher free fatty acid lipids usually cause symptoms within hours to days, while more inert lipids may take weeks to produce symptoms.

\section{Laboratory and roentgenographic findings}

It is possible to make the diagnosis of exogenous lipoid pneumonia only if all relevant data are known, which is more the exception than the rule. Because the disease may occur in an asymptomatic as well as a fulminant form, the blood count either may be normal or may reflect severe inflammation. Our patient, who was debilitated, had a WBC count of $20,000 / \mathrm{cu} \mathrm{mm}$, with a significant left differential shift. This could have been caused by the primary lipid reaction in the lung or by some other pneumonic process. Unless a secondary event, such as nausea, diarrhea, or dehydration, intervenes, serum electrolyte and other basic laboratory values will be in the normal range.

Especially in the early stages, the sputum specimen may be of no help or may actually provide the diagnosis. The sputum, if expectorated from deep in the lung, may show characteristic lipidladen macrophages, or it may reveal nothing at all. During a more chronic illness, a secondary bacterial infection may be present. Bronchoscopy may help to establish the diagnosis by providing a sample of sputum and biopsy specimen from a deeper region. More important, the procedure will not show cells typical of neoplasia or other specific disease. In these instances, the next step is needle or open lung biopsy.

Radiologically, the chest film may be clear or may show signs of fulminant illness. The patterns obtained on chest roentgenography will vary according to the amount of aspirate and the length of time that the lipid has been in the lung. Initially, air space consolidation will be seen. As the immunologic mechanism intervenes, the pattern may become interstitial as the macrophages leave the alveoli and enter the interstitium. The ability of the macrophages to handle the lipid will further determine the radiographic pattern. If the lipid is released into the alveoli, a combined alveolar and interstitial pattern may be produced. Atelectasis often is seen as the airways become consolidated. Later, as diffuse inflammation occurs, conglomerate fibrotic patterns may be produced; these patterns often simulate a tumor. ${ }^{4}$ As with most aspiration, the right lower lobe is affected most frequently, but any lobe may be involved. In many cases, the roentgenographic pattern appears much worse than the patient's clinical status, thus prompting investigation into the atypical pneumonias. Pleural effusions are present at times; these usually are found to be reactive and are of little help in diagnosis.

Because this pneumonia may appear to involve a tumor, computed tomography (CT) often is performed. According to Wheeler and associates, ${ }^{5}$ measurement of density may provide an important clue to the diagnosis. Mineral oil and fat have CT densities of -150 to -60 Hounsfield units.

Early pathologic findings include pneumonitis with thickened alveolar septa and possibly oil in the alveolar space. Later, oil or lipid-laden macrophages may be present in the alveoli and the interstitium. Sudan III (oil red) or Sudan black B fat stain will confirm the presence of lipid. Further chronic inflammatory changes and lymphoid hyperplasia are found as macrophages are broken down. Finally, necrosis and hemorrhage may occur in more extensive cases. Pathologic findings may be confused with those present in congenital lipidosis and histiocytosis X.6,7

The patient's age and the amount and duration or continuation of aspiration determine the degree of debilitation. Several authors have reported the severity of inflammation and overall lung damage to be associated with the type of lipid ingested. Genereux $^{7}$ and Kennedy and coworkers ${ }^{8}$ have found that vegetable and mineral oil produce a more simple foreign body reaction, whereas animal fats, because of their higher free fatty acid content, are more prone to cause necrosis and hemorrhage. Underlying disorders, such as bronchiectasis, emphysema, or asthma, would produce a more fulminant disease. Exogenous lipoid pneumonia also may be complicated by bacterial, viral, or fungal infection when defense mechanisms, including ciliary response and cellular defense, are compromised.

\section{Treatment}

The most important treatment for this illness includes removal of the offending agent and supportive care. Findings from their study led Ayvazian and colleagues ${ }^{9}$ to suggest that corticosteroid therapy merits further trial in diffuse pulmonary disease that is caused by aspirated lipid. Warson and associates ${ }^{10}$ stated that a complication of lipoid pneumonia, interstitial fibrosis, may be halted by cortisone, which stabilizes the macrophages in their attempt to rid the lung of lipid, then lyses and releases free radicals and other toxins.

Frequent sputum specimens should be obtained 
for culturing of secondary bacterial or fungal overgrowth, and appropriate antibiotics should be administered if necessary. If acute aspiration is suspected, therapeutic bronchoscopy and bronchoalveolar lavage may be of benefit in reducing the load of lipid that must be handled by the lung's defense mechanisms.

\section{Summary}

Exogenous lipoid pneumonia should be considered when a patient prone to aspiration has pneumonia or when the chest film does not contribute to the diagnosis. The illness can range from mild to severe and may be fatal. Early recognition and supportive care are the key to treatment and prevention of severe illness. Keeping this disease in the back of one's mind may unmask this great mimicker early.

1. Laughlen GF: Studies on pneumonia following naso-pharyngeal ingestion of oil. Am J Pathol 1925;1:407-414.
2. Miller A, Bader RA, Bader ME, et al: Mineral oil pneumonia. Ann Intern Med 1962;57:627-634.

3. Beerman B, Christensson T, Moller P, et al: Lipoid pneumonia: An occupational hazard of fire eaters. $B r$ Med $J$ 1984;289:1728-1729.

4. Lipinski JK, Weisbrod GL, Sanders DE: Exogenous lipoid pneumonitis: Pulmonary patterns. AJR 1981;136:931-934.

5. Wheeler PS, Stitik FP, Hutchins GM, et al: Diagnosis of lipoid pneumonia by computed tomography. JAMA 1981;245:65-66.

6. Borrie J, Gwynne JF: Paraffinoma of lung: Lipoid pneumonia. Report of two cases. Thorax 1973;28:214-221.

7. Genereux GP: Lipids in the lungs: Radiologic-pathologic correlation. $J$ Can Assoc Radiol 1970;21:2-15.

8. Kennedy JD, Costello P, Balikian JP, et al: Exogenous lipoid pneumonia. AJR 1981;136:1145-1149.

9. Ayvazian LF, Steward DS, Merkel CG, et al: Diffuse lipoid pneumonitis successfully treated with prednisone. Am J Med 1967;43:930-934.

10. Warson F, Segers D, Roels P: An unsuspected case of lipoid pneumonia: Report of a case and review of the literature. Acta Clin Belg $1984 ; 39: 368-373$

From the departments of internal medicine and pathology, Metropolitan Hospital-Springfield Division, Springfield, Penn.

Reprint requests to Dr Cornacchia, 196A Fairview Rd, Woodlyn, PA 19094. 


\section{This is what too many
of your colleagues are doing to treat drug abuse}

Many aren't even looking for the drug abuser.

Yet, people using illegal drugs are seeing their physicians all the time. Isn't it time we all looked closer?

Finding the drug abuser isn't always easy, but it's a beginning. 